\title{
Comparación de dos métodos para reconocimiento de dígitos manuscritos fuera de línea
}

\author{
María Cristina Guevara Neri ${ }^{1}$, Osslan Osiris Vergara Villegas ${ }^{1}$, \\ Vianey Guadalupe Cruz Sánchez ${ }^{1}$, Juan Humberto Sossa Azuela ${ }^{2,3}$ \\ ${ }^{1}$ Universidad Autónoma de Ciudad Juárez, Chihuahua, \\ México \\ ${ }^{2}$ Instituto Politécnico Nacional (CIC), \\ México \\ ${ }^{3}$ Instituto Tecnológico de Estudios Superiores Monterrey (Campus Guadalajara), \\ México
}

mc_guevara_neri@hotmail.com, \{overgara,vianey.cruz\}@uacj.mx, hsossa@cic.ipn.mx

\begin{abstract}
Resumen. En el presente artículo, se muestra el resultado de la comparación del desempeño de dos métodos para el reconocimiento de dígitos manuscritos fuera de línea. El primer método, es una red de perceptrones con la cual se clasificaron las imágenes tras realizar una comparación por pares de clases; el segundo, es un método novedoso que realiza una comparación pixel por pixel entre la imagen por clasificar, y las imágenes de referencia. Para las pruebas, se utilizó un subconjunto de 450 imágenes de la base de datos MNIST. Cada método fue evaluado en dos partes: primero, con un conjunto de 100 imágenes de entrenamiento, y segundo, con un conjunto de 350 imágenes de prueba. Con el primer clasificador se obtuvo una exactitud del $93.86 \%$, y con el segundo se consiguió una del $95.14 \%$. Después del análisis de los resultados obtenidos se demuestra que el segundo método se desempeñó mejor que el primero. La fortaleza del método novedoso radica principalmente en su robustez y tiempo de ejecución.
\end{abstract}

Palabras clave: Reconocimiento de dígitos manuscritos, red de perceptrones, comparación vector con vector, MNIST.

\section{Comparison of Two Off-Line Handwritten Digits Recognition Methods}

\begin{abstract}
In this paper, the results of the comparison between two off-line handwritten digits recognition methods are presented. The first method is a network of perceptrons with which the images were classified after making a comparison by pairs of classes; the second, is a new method that performs a pixel by pixel comparison between the image to be classified, and the reference images. For the tests, a subset of 450 images from the MNIST database was used.
\end{abstract}


María Cristina Guevara Neri, Osslan Osiris Vergara Villegas, Vianey Guadalupe Cruz Sánchez, et al.

Each method was evaluated in two parts: first, with a set of 100 training images, and second, with a set of 350 test images. With the first classifier, an accuracy of $93.86 \%$ was obtained, and with the second, an accuracy of $95.14 \%$. After the analysis of the results, it is shown that the second method outperformed the first. The strength of the new method lies mainly in its robustness and execution time.

Keywords: Handwritten digits recognition, perceptron network, comparison between vectors, MNIST.

\section{Introducción}

En la última década (2008-2018), se ha observado un incremento en la digitalización de documentos, por lo que, el reconocimiento de dígitos manuscritos, y caracteres en general, es un área de interés para científicos, académicos e industriales [1]. El reconocimiento de caracteres manuscritos ha ganado popularidad en el área de investigación, y ha generado el reto entre los investigadores de lograr emular el sistema de procesamiento visual humano, mediante el desarrollo de algoritmos computacionales.

El reconocimiento de dígitos manuscritos es una rama correspondiente a la tecnología de reconocimiento óptico de caracteres, en la que el reto consiste en cómo utilizar un dispositivo procesador para reconocer automáticamente los dígitos [2]. La lectura de caracteres manuscritos representa una tarea complicada para una máquina, ya que no cuenta, como lo hace un ser humano, con la posibilidad de tomar decisiones fuera de un esquema establecido.

Las técnicas de reconocimiento de dígitos manuscritos se clasifican en dos grandes áreas que son en línea y fuera de línea. En la primera técnica, el flujo de dígitos es reconocido en el momento en el que es escrito, mientras que, en la segunda, que suele ser más compleja, los dígitos son reconocidos mediante la captura o escaneo de imágenes. Usualmente, el reconocimiento de dígitos manuscritos consiste en las siguientes etapas: 1) adquisición, 2) pre-procesamiento, 3) segmentación, 4) extracción de características, y 5) clasificación [3].

La etapa de adquisición o digitalización consiste en adquirir la imagen de los dígitos manuscritos a través de una fotografía o escaneo. En el pre-procesamiento se aplican diversos algoritmos de mejoramiento con la finalidad de mejorar la calidad de la información. Por ejemplo, en las imágenes de dígitos manuscritos se puede presentar ruido debido al color del papel, la textura, imágenes de fondo, emborronamiento, distorsiones por la perspectiva de la imagen, variaciones en la iluminación, el cual debe ser eliminado en la medida de lo posible [4].

La segmentación de los objetos de la imagen es la fase donde se tiene como objetivo separar el área correspondiente al dígito, y el área del fondo [5]. En la extracción de características se transforma la información de entrada en descriptores esenciales para distinguir un dígito de otro [6]. Finalmente, la clasificación representa la predicción de la clase (o etiqueta) para un objeto, basado en la similitud del dígito contra los dígitos de referencia [7]. Uno de los algoritmos más simples de clasificación es el vecino más 
cercano (k-NN, del inglés k-Nearest Neighbor), el cual se basa en el uso de distancias (Euclidiana, Manhattan, Hamming, etc.) [8]. Por otro lado, las Redes Neuronales Artificiales (RNA) tienen como objetivo procesar información de la misma manera que el cerebro humano lo hace, por lo que cuentan con una fase de entrenamiento y aprendizaje y han sido muy utilizadas en la etapa de clasificación de dígitos manuscritos $[1,2,7]$. La técnica de Máquina con Vectores Soporte (SVM, del inglés Support Vector Machine), se basa en el descubrimiento de un hiperplano que se usa para separar los datos de las clases existentes [9]. En [10] se puede consultar un estudio de las diferentes técnicas de clasificación de dígitos manuscritos.

Aun cuando en la literatura se han presentado diversos trabajos sobre el reconocimiento de dígitos manuscritos, la tarea sigue representando un reto en el área de visión por computadora, debido a la diversidad de estilos de escritura asociados a diferentes personas, las herramientas utilizadas para escribir (pluma, lápiz), y la falta de cuidado al realizar los trazos. Por lo tanto, en el presente trabajo se realiza una comparación entre dos métodos de clasificación para el reconocimiento de dígitos manuscritos fuera de línea: un método fundamentado en el uso de una RNA de perceptrones, y un nuevo método basado en comparaciones similar al k-NN.

\section{Materiales y métodos}

La metodología para la clasificación de dígitos manuscritos utilizada en el presente artículo es similar a la explicada en la sección 1 y se puede observar en la Figura 1.

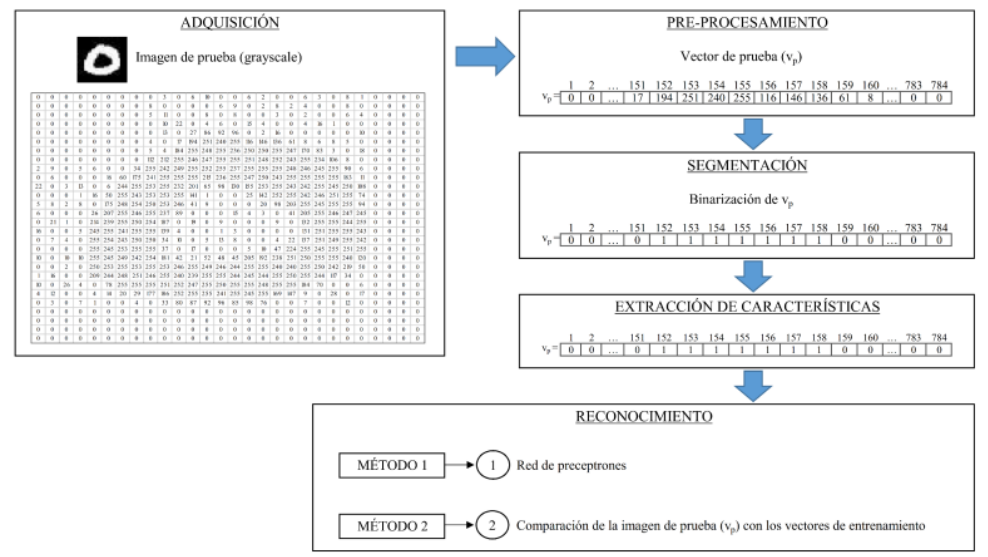

Fig. 1. Etapas propuestas para el reconocimiento de dígitos manuscritos

En la literatura sobre reconocimiento de dígitos manuscritos, existen diversas bases de datos tales como: The Street View House Numbers (SVHN) [11], The National Institute of Standards and Technology (NIST) Special Database 19 [12], y The Modified National Institute of Standards and Technology (MNIST) database [13, 14], que se han utilizado como estándar para probar la robustez de los métodos propuestos. Sin embargo, MNIST creada por Lecun [14], es la que más se utiliza en la literatura, 


\section{9 \\ 0123456789 0123456789 01123456789 01123456789}

Fig. 2. Ejemplo de algunos dígitos del subconjunto seleccionado de MNIST.

por lo que fue seleccionada en el presente artículo. La base de datos MNIST se forma por un conjunto de 60000 imágenes de entrenamiento y 10000 de prueba de dígitos manuscritos del 0 al 9, las cuales están normalizadas, es decir, cada dígito tiene un tamaño de 20 x 20 pixeles, y se encuentra sobre un fondo de 28 x 28 pixeles (centrado mediante el centro de gravedad). Para probar los dos métodos de clasificación del presente artículo, se seleccionó al azar un subconjunto de 450 imágenes, 100 para la fase de entrenamiento, 10 por cada dígito desde el 0 al 9, y 350 para la fase de pruebas, 35 por cada dígito desde el 0 al 9. En la Figura 2, se muestra un ejemplo de algunos de los dígitos seleccionados.

Cada una de las 450 imágenes fue preprocesada por medio de una transformación (reacomodo) que genera una representación vectorial de 1 x 784 píxeles. En seguida, cada vector (que se encuentra en escala de grises, con valores entre 0 y 255) es segmentado por medio de un proceso de binarización. Para binarizar las imágenes se aplicó la regla siguiente: para cada pixel de la imagen de entrada ( $I m)$, si el valor del pixel es menor que 100, que es el valor del umbral ${ }^{1}$, se le asigna un valor de 0 , y si el valor del pixel es mayor o igual que 100, entonces se le asigna un valor de 1 , como se puede observar en las Ecuaciones 1 y 2.

$$
\begin{aligned}
& \operatorname{Si} \operatorname{Im}(i, j)<100, \quad \operatorname{Im}(i, j)=0 \text { con } i, j \text { de } 1 \text { a 28, } \\
& \operatorname{Si} \operatorname{Im}(i, j) \geq 100, \quad \operatorname{Im}(i, j)=1 \text { con } i, j \text { de } 1 \text { a } 28 .
\end{aligned}
$$

Los 784 valores obtenidos del proceso de segmentación son utilizados como el vector de características para describir cada uno de los dígitos, cabe mencionar que en el presente trabajo no se realizó una etapa de selección de características. Finalmente, se realiza el reconocimiento de los dígitos por medio de dos métodos diferentes.

Antes de describir los métodos utilizados para clasificación es importante mencionar que: a) El primer método, utiliza un concepto conocido: el perceptrón, b) El segundo método, es un método nuevo propuesto en el presente trabajo, el cual consiste en la aplicación de una regla de comparación entre imágenes, c) Ambos métodos se aplicaron a los mismos conjuntos de imágenes, d) Los experimentos para ambos métodos se realizaron bajo las mismas condiciones.

\footnotetext{
${ }^{1}$ Después de un análisis exhaustivo donde se analizó el valor de cada pixel de la imagen, se obtuvo que el umbral 100 permite que todos los dígitos queden segmentados de manera adecuada.
} 


\subsection{Método 1: red de perceptrones}

El primer método utilizó una red de perceptrones para la clasificación de las imágenes. Un perceptrón es un algoritmo de aprendizaje binario que permite determinar si el dígito a clasificar pertenece o no a determinada clase [15].

Para crear la red, se utilizó la función perceptron incluida en la librería estándar de MATLAB, la cual emplea como base para la clasificación la función de transferencia hardlimit. La función perceptron requiere una fase de entrenamiento (train), y, utiliza el parámetro epoch, que es la aplicación de la regla de aprendizaje de la red a cada ejemplo en el conjunto de datos ( 1 epoch $=1$ aplicación de la regla). El método utilizó, en promedio, alrededor de 6 epochs por perceptrón.

Como el perceptrón realiza la clasificación entre dos tipos de clases, se construyó una red de 90 perceptrones para poder realizar todas las comparaciones entre la imagen de prueba y las 10 clases posibles como se muestra en la Figura 3.

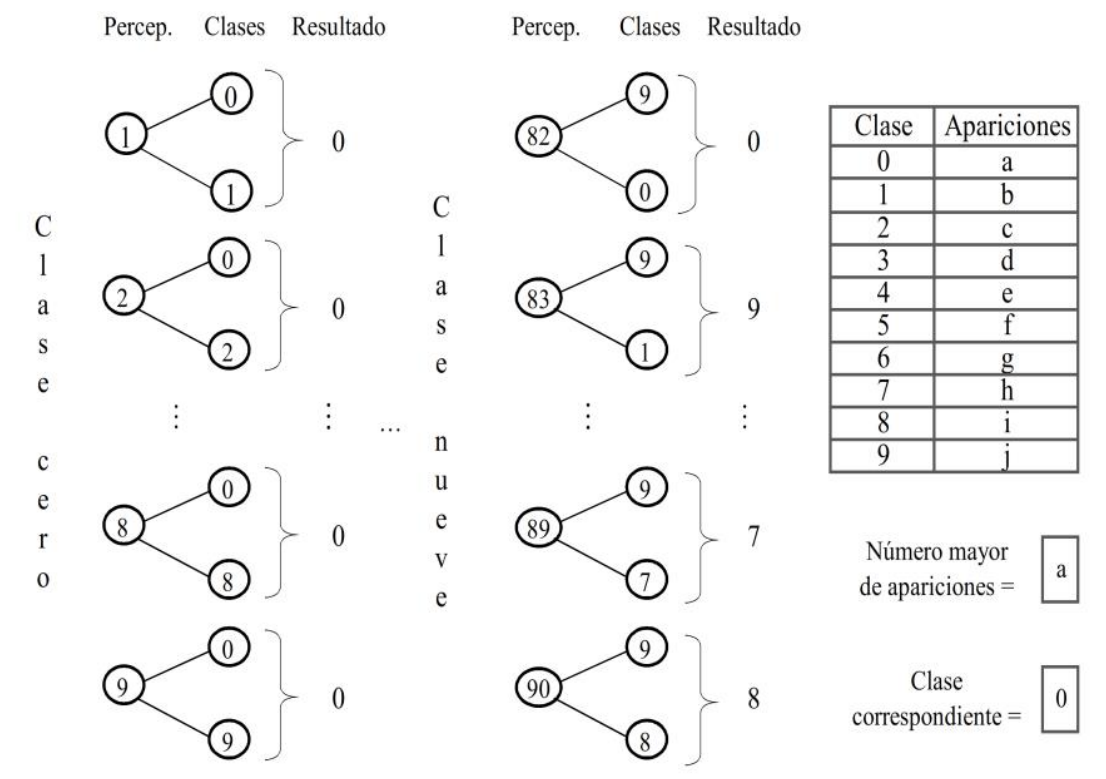

Fig. 3. Red de perceptrones utilizada en el método 1.

Las comparaciones para decidir cuál era la clase a la que más se parecía la imagen de prueba se realizaron por pares, y finalmente, se realizó la clasificación con la clase más repetida en todas las comparaciones hechas. La Tabla 1 muestra un ejemplo con una imagen de prueba correspondiente a la clase cero.

Cada renglón de la Tabla 1 corresponde a dos perceptrones, donde las columnas referentes a las clases indican cuáles fueron las clases analizadas (por pares), y la columna de clasificación muestra cuál fue el resultado obtenido. En la Tabla 2, se muestra el número de apariciones de cada una de las clases predichas por el clasificador para la imagen de la Tabla 1. 
María Cristina Guevara Neri, Osslan Osiris Vergara Villegas, Vianey Guadalupe Cruz Sánchez, et al.

Tabla 1. Clasificación de una imagen de prueba correspondiente a un cero.

\begin{tabular}{|c|c|c|c|c|c|}
\hline Clase 1 & Clase 2 & Clasificación & Clase 1 & Clase 2 & Clasificación \\
\hline cero & uno & cero & cinco & cero & cero \\
\hline cero & dos & cero & cinco & uno & cinco \\
\hline cero & tres & cero & cinco & dos & cinco \\
\hline cero & cuatro & cero & cinco & tres & tres \\
\hline cero & cinco & cero & cinco & cuatro & cinco \\
\hline cero & seis & cero & cinco & seis & cinco \\
\hline cero & siete & cero & cinco & siete & cinco \\
\hline cero & ocho & cero & cinco & ocho & cinco \\
\hline cero & nueve & cero & cinco & nueve & cinco \\
\hline uno & cero & cero & seis & cero & cero \\
\hline uno & dos & dos & seis & uno & seis \\
\hline uno & tres & tres & seis & dos & seis \\
\hline uno & cuatro & cuatro & seis & tres & tres \\
\hline uno & cinco & cinco & seis & cuatro & cuatro \\
\hline uno & seis & seis & seis & cinco & cinco \\
\hline uno & siete & siete & seis & siete & siete \\
\hline uno & ocho & ocho & seis & ocho & ocho \\
\hline uno & nueve & nueve & seis & nueve & seis \\
\hline dos & cero & cero & siete & cero & cero \\
\hline dos & uno & dos & siete & uno & siete \\
\hline dos & tres & tres & siete & dos & dos \\
\hline dos & cuatro & dos & siete & tres & tres \\
\hline dos & cinco & cinco & siete & cuatro & cuatro \\
\hline dos & seis & seis & siete & cinco & siete \\
\hline dos & siete & dos & siete & seis & siete \\
\hline dos & ocho & dos & siete & ocho & ocho \\
\hline dos & nueve & dos & siete & nueve & siete \\
\hline tres & cero & cero & ocho & cero & cero \\
\hline tres & uno & tres & ocho & uno & ocho \\
\hline tres & dos & tres & ocho & dos & dos \\
\hline tres & cuatro & tres & ocho & tres & tres \\
\hline tres & cinco & cinco & ocho & cuatro & ocho \\
\hline tres & seis & tres & ocho & cinco & cinco \\
\hline tres & siete & tres & ocho & seis & ocho \\
\hline tres & ocho & tres & ocho & siete & ocho \\
\hline tres & nueve & tres & ocho & nueve & ocho \\
\hline cuatro & cero & cero & nueve & cero & cero \\
\hline cuatro & uno & cuatro & nueve & uno & nueve \\
\hline cuatro & dos & dos & nueve & dos & dos \\
\hline cuatro & tres & tres & nueve & tres & tres \\
\hline cuatro & cinco & cinco & nueve & cuatro & cuatro \\
\hline cuatro & seis & cuatro & nueve & cinco & cinco \\
\hline cuatro & siete & cuatro & nueve & seis & nueve \\
\hline cuatro & ocho & cuatro & nueve & siete & siete \\
\hline cuatro & nueve & cuatro & nueve & ocho & ocho \\
\hline
\end{tabular}


Comparación de dos métodos para reconocimiento de dígitos manuscritos fuera de línea

Tabla 2. Resumen del número total de aparición de clases para la imagen de la Tabla 1.

\begin{tabular}{cccc}
\hline Clase & Apariciones & Clase & Apariciones \\
\hline cero & 18 & cinco & 14 \\
\hline uno & 0 & seis & 5 \\
\hline dos & 10 & siete & 7 \\
\hline tres & 15 & ocho & 9 \\
\hline cuatro & 9 & nueve & 3 \\
\hline
\end{tabular}

Como se puede observar en la Tabla 2, la clase que más se predijo fue la clase cero, con 18 apariciones (respecto al total de los 90 resultados), por lo cual la imagen fue clasificada como un cero. Cabe mencionar que en ninguna de las pruebas se presentó algún caso de empate entre el número mayor de apariciones de las clases, por lo que no se generó un criterio de desempate.

\subsection{Método 2: comparación vector contra vector}

El segundo método consiste en la aplicación de un nuevo algoritmo de comparación creado en MATLAB. El método se basa, como su nombre lo indica, en una comparación directa entre el vector de prueba $\left(v_{p}\right)$ y los vectores de entrenamiento $\left(v_{e}\right)$. Los vectores de entrenamiento se componen por 10 vectores renglón por dígito, cada uno de ellos correspondiente a una imagen, conformando así 100 vectores de entrenamiento en total. El vector de prueba corresponde al vector renglón que representa la imagen que se quiere clasificar. Es importante aclarar que los vectores de entrenamiento hacen referencia a los vectores que se están tomando como punto de referencia para la comparación que realiza el método, por lo tanto, el método propuesto al igual que un k-NN no tiene una fase de aprendizaje, además el método propuesto no necesita una medida de distancia.

El vector de prueba es comparado contra cada uno de los vectores de entrenamiento, es decir, cada una de sus componentes se contrasta contra cada una de las componentes de los 100 vectores, para calcular a cuál se parece más. Por lo que, el número de comparaciones hechas por cada imagen de prueba que se compara, contra cada clase, es de 78400 (784 componentes del vector de prueba por 100 vectores de entrenamiento). En cada comparación, se asigna un valor específico que se obtiene de la siguiente manera: por cada componente correspondiente de los vectores que sea exactamente igual, a la comparación se le fija un valor 0 , y por cada que sea distinta se le fija un valor 1, como se muestra en las Ecuaciones 3 y 4.

$$
\begin{aligned}
& \text { Si } v_{p}(1, i)=v_{e}(1, i), \quad a=0 \text { con } i \text { de } 1 \text { a 784, } \\
& \text { Si } v_{p}(1, i) \neq v_{e}(1, i), \quad a=1 \text { con } i \text { de } 1 \text { a } 784 \text {. }
\end{aligned}
$$

Finalmente, todos los valores de cada comparación entre componentes se suman y generan como resultado un valor de comparación final, el cual se almacena en un vector (z), como se muestra en la Ecuación 5, de manera que cuando se realizan las 100 comparaciones, se selecciona aquella con el valor más pequeño (ver Ecuación 6), y se le determina una clase del cero al nueve, de acuerdo con la posición del valor mínimo. 
Cabe mencionar que en las pruebas realizadas no se presentó algún caso de empate entre los valores más pequeños, por lo que no se generó un criterio de desempate.

$$
\begin{gathered}
z(j, 1)=\sum_{i=1}^{784} a_{i}, \operatorname{con} j \text { de } 1 \text { a } 100, \\
\text { Clase } \leftarrow \min (z) .
\end{gathered}
$$

En la Figura 4 se muestra de manera visual un ejemplo del funcionamiento del nuevo método propuesto.

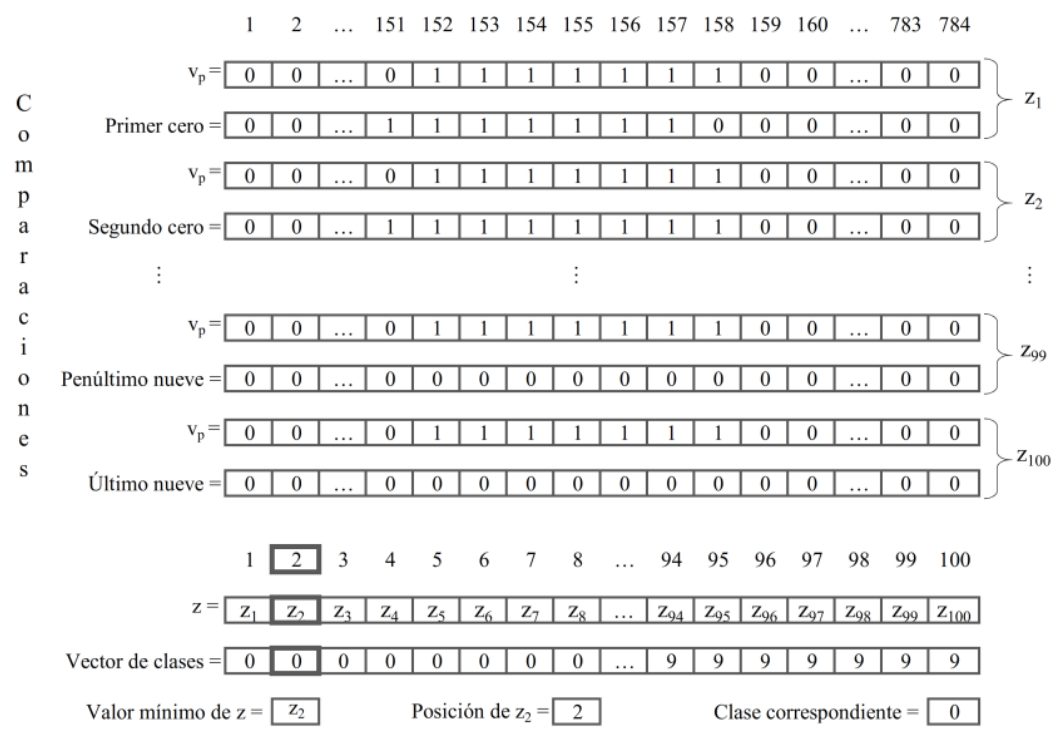

Fig. 4. Funcionamiento del método de comparación de vector contra vector.

\section{Experimentación y resultados}

Para cada uno de los métodos se realizaron experimentos con los mismos dos conjuntos de imágenes: 1) entrenamiento (100), y 2) prueba (350). En ambos casos se presentan los resultados del entrenamiento y de la prueba. Todas las pruebas se realizaron en una laptop HP con procesador Intel Core i5 con memoria RAM de 8Gb y las implementaciones de los dos métodos se realizaron con MATLAB.

La Tabla 3 muestra la notación, fórmula y descripción de las variables medidas. Es importante aclarar que el comportamiento de ambos métodos a lo largo de los experimentos siempre fue el mismo, por lo que los datos presentados en las siguientes subsecciones corresponden a una única corrida por imagen. 
Comparación de dos métodos para reconocimiento de dígitos manuscritos fuera de línea

\subsection{Resultados obtenidos con el método 1}

Tabla 3. Variables de medición obtenidas de [16].

\begin{tabular}{ccl}
\hline Variable (notación) & Fórmula & \multicolumn{1}{c}{ Descripción } \\
\hline $\begin{array}{c}\text { Verdaderos } \\
\text { Positivos (VP) } \\
\begin{array}{c}\text { Verdaderos } \\
\text { Negativos (VN) }\end{array}\end{array}$ & - & Casos que fueron predichos SÍ, y realmente fueron SÍ \\
\hline $\begin{array}{c}\text { Falsos } \\
\text { Negativos (FN) }\end{array}$ & - & Casos que fueron predichos NO, y realmente fueron NO fueron predichos NO, y realmente fueron SÍ \\
\hline $\begin{array}{c}\text { Falsos } \\
\text { Positivos (FP) }\end{array}$ & - & Casos que fueron predichos SÍ, y realmente fueron NO \\
\hline Exactitud & (VP+VN) / total & En general, ¿qué tan frecuente el clasificador acierta? \\
\hline Tasa de error & (FP+FN) / total & En general, ¿qué tan frecuente el clasificador NO acierta? \\
\hline Sensibilidad & VP / SÍ real & $\begin{array}{l}\text { Cuando es un SÍ real, ¿qué tan frecuente el clasificador pre- } \\
\text { dice un SÍ? }\end{array}$ \\
\hline Especificidad & VN / NO real & $\begin{array}{l}\text { Cuando es un NO real, ¿qué tan frecuente el clasificador pre- } \\
\text { dice un NO? }\end{array}$ \\
\hline Precisión & VP / SÍ predicho & $\begin{array}{l}\text { Cuando el clasificador predice SÍ, ¿qué tan frecuente está en } \\
\text { lo correcto? }\end{array}$ \\
\hline
\end{tabular}

Tabla 4. Método 1: resultados obtenidos con 100 imágenes de entrenamiento.

\begin{tabular}{rccccccccccc}
\hline & \multicolumn{10}{c}{ CLASE } \\
\hline Variables & $\mathbf{0}$ & $\mathbf{1}$ & $\mathbf{2}$ & $\mathbf{3}$ & $\mathbf{4}$ & $\mathbf{5}$ & $\mathbf{6}$ & $\mathbf{7}$ & $\mathbf{8}$ & $\mathbf{9}$ & Total \\
\hline VP & 9 & 10 & 10 & 10 & 10 & 10 & 10 & 10 & 10 & 10 & 99 \\
\hline VN & 90 & 90 & 90 & 90 & 90 & 89 & 90 & 90 & 90 & 90 & - \\
\hline FP & 0 & 0 & 0 & 0 & 0 & 1 & 0 & 0 & 0 & 0 & - \\
\hline FN & 1 & 0 & 0 & 0 & 0 & 0 & 0 & 0 & 0 & 0 & 1 \\
\hline SÍ reales & 10 & 10 & 10 & 10 & 10 & 10 & 10 & 10 & 10 & 10 & 100 \\
\hline NO reales & 90 & 90 & 90 & 90 & 90 & 90 & 90 & 90 & 90 & 90 & - \\
\hline SÍ predichos & 9 & 10 & 10 & 10 & 10 & 11 & 10 & 10 & 10 & 10 & - \\
\hline NO predichos & 91 & 90 & 90 & 90 & 90 & 89 & 90 & 90 & 90 & 90 & - \\
\hline Variables & $\mathbf{0}$ & $\mathbf{1}$ & $\mathbf{2}$ & $\mathbf{3}$ & $\mathbf{4}$ & $\mathbf{5}$ & $\mathbf{6}$ & $\mathbf{7}$ & $\mathbf{8}$ & $\mathbf{9}$ & Media \\
\hline Exactitud & 0.9900 & 1.0000 & 1.0000 & 1.0000 & 1.0000 & 0.9900 & 1.0000 & 1.0000 & 1.0000 & 1.0000 & 0.9980 \\
\hline Tasa de error & 0.0100 & 0.0000 & 0.0000 & 0.0000 & 0.0000 & 0.0100 & 0.0000 & 0.0000 & 0.0000 & 0.0000 & 0.0020 \\
\hline Sensibilidad & 0.9000 & 1.0000 & 1.0000 & 1.0000 & 1.0000 & 1.0000 & 1.0000 & 1.0000 & 1.0000 & 1.0000 & 0.9900 \\
\hline Especificidad & 1.0000 & 1.0000 & 1.0000 & 1.0000 & 1.0000 & 0.9889 & 1.0000 & 1.0000 & 1.0000 & 1.0000 & 0.9989 \\
\hline Precisión & 1.0000 & 1.0000 & 1.0000 & 1.0000 & 1.0000 & 0.9091 & 1.0000 & 1.0000 & 1.0000 & 1.0000 & 0.9909 \\
\hline
\end{tabular}

Primero, se midió la capacidad para aprender del método 1. Los resultados obtenidos con las 100 imágenes de entrenamiento se muestran en la Tabla 4.

Los resultados obtenidos al aplicar el método 1 a las 350 imágenes de prueba se muestran en la Tabla 5. 
María Cristina Guevara Neri, Osslan Osiris Vergara Villegas, Vianey Guadalupe Cruz Sánchez, et al.

Tabla 5. Método 1: resultados obtenidos con 350 imágenes de prueba.

\begin{tabular}{|c|c|c|c|c|c|c|c|c|c|c|c|}
\hline \multirow[b]{2}{*}{ Variables } & \multicolumn{10}{|c|}{ CLASE } & \multirow[b]{2}{*}{ Total } \\
\hline & $\mathbf{0}$ & 1 & 2 & 3 & 4 & 5 & 6 & 7 & 8 & 9 & \\
\hline VP & 31 & 31 & 18 & 27 & 27 & 21 & 23 & 23 & 22 & 19 & 242 \\
\hline VN & 308 & 314 & 303 & 308 & 305 & 289 & 302 & 307 & 298 & 309 & - \\
\hline FP & 7 & 1 & 12 & 7 & 10 & 26 & 13 & 8 & 17 & 6 & - \\
\hline FN & 4 & 4 & 17 & 8 & 8 & 14 & 12 & 12 & 13 & 16 & 108 \\
\hline SÍ reales & 35 & 35 & 35 & 35 & 35 & 35 & 35 & 35 & 35 & 35 & 350 \\
\hline NO reales & 315 & 315 & 315 & 315 & 315 & 315 & 315 & 315 & 315 & 315 & - \\
\hline Sí predichos & 38 & 32 & 30 & 34 & 37 & 47 & 36 & 31 & 39 & 25 & - \\
\hline NO predichos & 312 & 318 & 320 & 316 & 313 & 303 & 314 & 319 & 311 & 325 & - \\
\hline Variables & $\mathbf{0}$ & 1 & 2 & 3 & 4 & 5 & 6 & 7 & 8 & 9 & Media \\
\hline Exactitud & 0.9686 & 0.9857 & 0.9171 & 0.9571 & 0.9486 & 0.8857 & 0.9286 & 0.9429 & 0.9143 & 0.9371 & 0.9386 \\
\hline Tasa de error & 0.0314 & 0.0143 & 0.0829 & 0.0429 & 0.0514 & 0.1143 & 0.0714 & 0.0571 & 0.0857 & 0.0629 & 0.0614 \\
\hline Sensibilidad & 0.8857 & 0.8857 & 0.5143 & 0.7714 & 0.7714 & 0.6000 & 0.6571 & 0.6571 & 0.6286 & 0.5429 & 0.6914 \\
\hline Especificidad & 0.9778 & 0.9968 & 0.9619 & 0.9778 & 0.9683 & 0.9175 & 0.9587 & 0.9746 & 0.9460 & 0.9810 & 0.9660 \\
\hline Precisión & 0.8158 & 0.9688 & 0.6000 & 0.7941 & 0.7297 & 0.4468 & 0.6389 & 0.7419 & 0.5641 & 0.7600 & 0.7060 \\
\hline
\end{tabular}

Tabla 6. Método 2: resultados obtenidos con 100 imágenes de entrenamiento.

\begin{tabular}{rccccccccccc} 
& \multicolumn{10}{c}{ CLASE } & \\
\cline { 2 - 10 } Variables & $\mathbf{0}$ & $\mathbf{1}$ & $\mathbf{2}$ & $\mathbf{3}$ & $\mathbf{4}$ & $\mathbf{5}$ & $\mathbf{6}$ & $\mathbf{7}$ & $\mathbf{8}$ & $\mathbf{9}$ & Total \\
\hline VP & 10 & 10 & 10 & 10 & 10 & 10 & 10 & 10 & 10 & 10 & 100 \\
VN & 90 & 90 & 90 & 90 & 90 & 90 & 90 & 90 & 90 & 90 & - \\
FP & 0 & 0 & 0 & 0 & 0 & 0 & 0 & 0 & 0 & 0 & - \\
FN & 0 & 0 & 0 & 0 & 0 & 0 & 0 & 0 & 0 & 0 & 0 \\
Sí reales & 10 & 10 & 10 & 10 & 10 & 10 & 10 & 10 & 10 & 10 & 100 \\
NO reales & 90 & 90 & 90 & 90 & 90 & 90 & 90 & 90 & 90 & 90 & - \\
Sí predichos & 10 & 10 & 10 & 10 & 10 & 10 & 10 & 10 & 10 & 10 & - \\
NO predichos & 90 & 90 & 90 & 90 & 90 & 90 & 90 & 90 & 90 & 90 & - \\
\hline Variables & $\mathbf{0}$ & $\mathbf{1}$ & $\mathbf{2}$ & $\mathbf{3}$ & $\mathbf{4}$ & $\mathbf{5}$ & $\mathbf{6}$ & $\mathbf{7}$ & $\mathbf{8}$ & $\mathbf{9}$ & Media \\
\hline Exactitud & 1.0000 & 1.0000 & 1.0000 & 1.0000 & 1.0000 & 1.0000 & 1.0000 & 1.0000 & 1.0000 & 1.0000 & 1.0000 \\
Tasa de error & 0.0000 & 0.0000 & 0.0000 & 0.0000 & 0.0000 & 0.0000 & 0.0000 & 0.0000 & 0.0000 & 0.0000 & 0.0000 \\
Sensibilidad & 1.0000 & 1.0000 & 1.0000 & 1.0000 & 1.0000 & 1.0000 & 1.0000 & 1.0000 & 1.0000 & 1.0000 & 1.0000 \\
Especificidad & 1.0000 & 1.0000 & 1.0000 & 1.0000 & 1.0000 & 1.0000 & 1.0000 & 1.0000 & 1.0000 & 1.0000 & 1.0000 \\
Precisión & 1.0000 & 1.0000 & 1.0000 & 1.0000 & 1.0000 & 1.0000 & 1.0000 & 1.0000 & 1.0000 & 1.0000 & 1.0000 \\
\hline
\end{tabular}

\subsection{Resultados obtenidos con el método 2}

Los resultados obtenidos al aplicar el método 2 a las 100 imágenes de entrenamiento se muestran en la Tabla 6.

Los resultados obtenidos al aplicar el método 2 a las 350 imágenes de prueba se muestran en la Tabla 7. 
Comparación de dos métodos para reconocimiento de dígitos manuscritos fuera de línea

Tabla 7. Método 2: resultados obtenidos con 350 imágenes de prueba.

\begin{tabular}{|c|c|c|c|c|c|c|c|c|c|c|c|}
\hline \multirow[b]{2}{*}{ VARIABLES } & \multicolumn{10}{|c|}{ CLASE } & \multirow[b]{2}{*}{ TOTAL } \\
\hline & $\mathbf{0}$ & 1 & 2 & 3 & 4 & 5 & 6 & 7 & 8 & 9 & \\
\hline VP & 33 & 34 & 22 & 23 & 28 & 23 & 28 & 27 & 24 & 23 & 265 \\
\hline $\mathrm{VN}$ & 305 & 301 & 312 & 310 & 308 & 304 & 310 & 303 & 312 & 300 & - \\
\hline FP & 10 & 14 & 3 & 5 & 7 & 11 & 5 & 12 & 3 & 15 & - \\
\hline FN & 2 & 1 & 13 & 12 & 7 & 12 & 7 & 8 & 11 & 12 & 85 \\
\hline SÍ reales & 35 & 35 & 35 & 35 & 35 & 35 & 35 & 35 & 35 & 35 & 350 \\
\hline NO reales & 315 & 315 & 315 & 315 & 315 & 315 & 315 & 315 & 315 & 315 & - \\
\hline SÍ predichos & 43 & 48 & 25 & 28 & 35 & 34 & 33 & 39 & 27 & 38 & - \\
\hline NO predichos & 307 & 302 & 325 & 322 & 315 & 316 & 317 & 311 & 323 & 312 & - \\
\hline VARIABLES & $\mathbf{0}$ & 1 & 2 & 3 & 4 & 5 & 6 & 7 & 8 & 9 & MEDIA \\
\hline Exactitud & 0.9657 & 0.9571 & 0.9543 & 0.9514 & 0.9600 & 0.9343 & 0.9657 & 0.9429 & 0.9600 & 0.9229 & 0.9514 \\
\hline Tasa de error & 0.0343 & 0.0429 & 0.0457 & 0.0486 & 0.0400 & 0.0657 & 0.0343 & 0.0571 & 0.0400 & 0.0771 & 0.0486 \\
\hline Sensibilidad & 0.9429 & 0.9714 & 0.6286 & 0.6571 & 0.8000 & 0.6571 & 0.8000 & 0.7714 & 0.6857 & 0.6571 & 0.7571 \\
\hline Especificidad & 0.9683 & 0.9556 & 0.9905 & 0.9841 & 0.9778 & 0.9651 & 0.9841 & 0.9619 & 0.9905 & 0.9524 & 0.9730 \\
\hline Precisión & 0.7674 & 0.7083 & 0.8800 & 0.8214 & 0.8000 & 0.6765 & 0.8485 & 0.6923 & 0.8889 & 0.6053 & 0.7689 \\
\hline
\end{tabular}

\subsection{Discusión}

De acuerdo con los resultados obtenidos con el primer conjunto de imágenes (de entrenamiento) mostrados en las Tablas 4 y 6 , se observa que el método 2 presentó un mejor desempeño, ya que clasificó correctamente todos los casos, mientras que el método 1 clasificó correctamente 99 de las 100 imágenes. Aunque los resultados arrojados por el método 1 son (relativamente) buenos, no son los esperados, ya que se esperaba que pudieran ser reconocidas todas las imágenes del conjunto, tal como lo hizo el método 2.

Con los resultados obtenidos en los experimentos hechos sobre el conjunto de imágenes de prueba mostrados en las Tablas 5 y 7 , se observa que el método 2 es mejor que el método 1. La comparación de las variables obtenidas mediante la aplicación de ambos métodos se muestra en la Tabla 8.

Tabla 8. Comparación de los métodos.

\begin{tabular}{ccc}
\hline Variables & Método 1 & Método 2 \\
\hline Exactitud & 0.9386 & 0.9514 \\
\hline Tasa de error & 0.0614 & 0.0486 \\
\hline Sensibilidad & 0.6914 & 0.7571 \\
\hline Especificidad & 0.9660 & 0.9730 \\
\hline Precisión & 0.7060 & 0.7689 \\
\hline
\end{tabular}

Como se puede observar en la Tabla 8, con el método 1 se obtuvo una sensibilidad de $69 \%$, es decir, clasificó de manera correcta 242 de las 350 imágenes, mientras que con el método 2 se obtuvo una sensibilidad de 75\%, es decir, de las 350 imágenes, clasificó correctamente 265. De igual forma, el método 2 presentó mejores resultados en exactitud, pues el clasificador fue mejor al momento de clasificar de manera general 


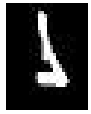

1: $6 / 1$

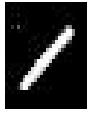

$1: 8 / 1$

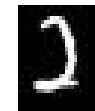

$2: 3 / 3$

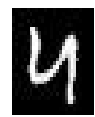

4: $4 / 4$

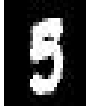

5: $5 / 5$

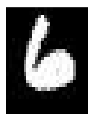

6: $4 / 6$

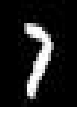

7:7/7

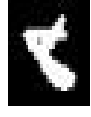

8: $5 / 8$

Fig. 5. Ejemplos de resultados para las imágenes de prueba.

las imágenes, es decir, acertó de manera más frecuente sobre cuándo una imagen pertenece a una clase, y cuándo no pertenece a una clase.

La Figura 5 ilustra algunos ejemplos tomados del conjunto de imágenes de prueba, con su respectiva clasificación (se debe observar que existen dígitos que a simple vista son difíciles de reconocer). El número a la izquierda (en negritas) representa la clase correcta a la que pertenece la imagen, los siguientes dos números refieren a la clase dada por ambos clasificadores, (método 1/método 2).

Aunque los resultados entre los dos métodos se encuentran relativamente cerca, la principal diferencia radica en el tiempo de ejecución. El método 2 fue significativamente más rápido para la prueba y entrenamiento, en comparación con el método 1. Para crear una idea sobre qué tan significativa fue la diferencia respecto a los tiempos de ejecución, considere lo siguiente: el método 2 tardó en clasificar las 450 imágenes en un tiempo menor a $10 \mathrm{~s}$, mientras que el método 1 tardó en clasificar las mismas imágenes, y bajo las mismas condiciones, alrededor de $90 \mathrm{~min}$. Por lo que se si se utilizaran, por ejemplo, 42000 imágenes de MNIST, el método 2 terminaría de clasificarlas todas, en el mismo tiempo que el método 1 solamente clasificaría 70.

Con respecto a la bondad del método presentado contra los trabajos de la literatura se observa que es difícil realizar una comparación real. Aun cuando existen trabajos que utilizan MNIST, normalmente, no se utiliza el mismo subconjunto de imágenes y se tendrían que programar los otros métodos. Sin embargo, para el caso de la clasificación nuestro trabajo obtuvo una exactitud de 0.9514 con el nuevo método propuesto, mientras que en el trabajo de [2], se utilizó una RNA de pico de descenso aproximado normalizado y se obtuvo una exactitud de 0.9817; en el trabajo de [5], se utilizó una SVM y se obtuvo una exactitud de 0.9691, en la investigación de [7], se utilizó una red de creencias profundas con aprendizaje Q y se obtuvo una exactitud de 0.9918 , además se reporta un tiempo de $21.46 \mathrm{~s}$ para la clasificación de 100 imágenes de MNIST. Finalmente, en el trabajo presentado en [9], se utilizó un híbrido de una SVM y una red neuronal convolucional en la que se reporta una exactitud de 0.9981 . Como se puede observar, el método 2 presentado en este artículo obtuvo resultados de exactitud competitivos contra los presentados en el estado del arte y además buen tiempo de ejecución, con la característica adicional que nuestro método es computacionalmente menos complejo.

\section{Conclusiones}

Se presentó una comparación entre dos métodos para el reconocimiento de dígitos manuscritos fuera de línea. Para poder realizar una comparación justa para ambos métodos se utilizó un subconjunto de 450 imágenes de MNIST. En el método 1 se implementó una red de perceptrones, mientras que en el método 2 se presentó un nuevo 
algoritmo que consiste en la comparación entre las características de imágenes de prueba y las características de imágenes de referencia similar al k-NN con la diferencia de que no se calculan distancias.

De acuerdo con los resultados obtenidos se concluye que el nuevo método propuesto (2) tiene un mejor desempeño comparado con el método 1. La conclusión se obtiene a partir de la eficiencia del clasificador al realizar la clasificación con los diversos conjuntos de imágenes, así como el tiempo de ejecución que utiliza cada método para realizar la tarea asignada. Además de la eficiencia en la clasificación es importante destacar que, el método propuesto en el presente trabajo está basado en el uso de operaciones de comparación, en donde se conoce qué está siendo comparado, y cómo se realiza la comparación, mientras que en el método 1 no ocurre lo mismo, puesto que al utilizar el perceptrón se conoce qué se está clasificando, pero no cómo está siendo clasificado.

Como trabajo futuro se considera: aumentar el tamaño del conjunto de imágenes de entrenamiento y de prueba, y modificar el método 1 para reducir el tiempo de ejecución, a través de la reducción del número de perceptrones utilizados, mediante el descarte temprano de algunas clases. Además, será importante realizar pruebas con otros tipos de clasificadores.

Agradecimientos. H. Sossa agradece al Instituto Politécnico Nacional y al CONACYT, a través de los apoyos económicos en el marco de fondos SIP 20180730 y 65 (Fronteras de la Ciencia). María Cristina Guevara Neri agradece al CONACYT por la beca otorgada para la realización de sus estudios de doctorado.

\section{Referencias}

1. Kulkarni, S., Rajendran, B.: Spiking neural networks for handwritten digit recognitionsupervised learning and network optimization. Neural Networks, 103, pp.118-127 (2018)

2. Sueiras, J., Ruiz, V., Sanchez, A., Velez, J.: Offline continuous handwriting recognition using sequence to sequence neural networks. Neurocomputing, 289, pp. 119-128 (2018)

3. Kumar, M., Jindal, M., Sharma, R., Rani, S.: Character and numeral recognition for nonIndic and Indic scripts: A survey. Artificial Intelligence Review, pp. 1-27 (2018)

4. Mandal, S., Mahadeva, S., Sundaram, S.: GMM posterior features for improving online handwriting recognition. Expert Systems with Applications, 97, pp. 421-433 (2018)

5. Gattal, A., Chibani, Y., Hadjadji, B.: Segmentation and recognition system for unknownlength handwritten digit strings. Pattern Analysis and Applications. 20(2), pp. 307-323 (2017)

6. Surinta, O., Karaaba, M., Schomaker, L., Wiering, M.: Recognition of handwritten characters using local gradient feature descriptors. Engineering Applications of Artificial Intelligence, 45, pp. 405-414 (2015)

7. Qiao, J., Wang, G., Li, W., Chen, M.: An adaptive deep Q-learning strategy for handwritten digit recognition. Neural Networks. Article in Press, pp. 1-18 (2018)

8. Bhattacharya, G., Ghosh, K., Chowdhury, A.: An affinity-based new local distance function and similarity measure for kNN algorithm. Pattern Recognition Letters. 33(3), pp. 356-363 (2012)

9. Niu, X., Suen, C.: A novel hybrid CNN-SVM classifier for recognizing handwritten digits. Pattern Recognition, 45(4), pp. 1318-1325 (2012) 
María Cristina Guevara Neri, Osslan Osiris Vergara Villegas, Vianey Guadalupe Cruz Sánchez, et al.

10. Al-Helali, B., Mahmoud, S.: Arabic online handwriting recognition (AOHR): A survey. ACM Computing Surveys, 50(3), pp. 1-35 (2017)

11. The Street View House Numbers (SVHN) Dataset: http://ufldl.stanford.edu/housenumbers/, last accessed (2018)

12. NIST: National Institute of Standards and Technology. NIST Special Database 19 (2018)

13. The MNIST database: http://yann.lecun.com/exdb/mnist/ (2018)

14. LeCun, Y., Jackel, L., Bottou, L., Cortes, C., Denker, J., Drucker, H., Guyon, I., Muller, A., Sackinger, E., Simard, P., Vapnik, V.: Learning algorithms for classification: A comparison on handwritten digit recognition. Neural Networks: The Statistical Mechanics Perspective, pp. 261-276 (1995)

15. Kurkova, V., Sanguineti, M.: Probabilistic lower bounds for approximation by shallow perceptron networks. Neural Networks, 91, pp. 31-41 (2017)

16. Markham, K.: Simple guide to confusion matrix terminology, http://www.dataschool.io/simple-guide-to-confusion-matrix-terminology/ (2018) 